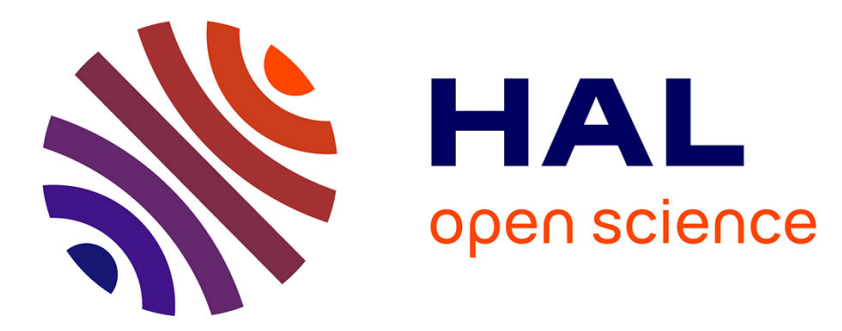

\title{
Ice-templated porous tungsten and tungsten carbide inspired by natural wood
}

Yuan Zhang, Guoqi Tan, Da Jiao, Jian Zhang, Shaogang Wang, Feng Liu, Longchao Zhuo, Zhefeng Zhang, Sylvain Deville, Robert O Ritchie, et al.

\section{- To cite this version:}

Yuan Zhang, Guoqi Tan, Da Jiao, Jian Zhang, Shaogang Wang, et al.. Ice-templated porous tungsten and tungsten carbide inspired by natural wood. JOURNAL OF MATERIALS SCIENCE \& TECHNOLOGY, 2020, 45, pp.187-197. 10.1016/j.jmst.2019.10.021 . hal-02441780

\author{
HAL Id: hal-02441780 \\ https://hal.science/hal-02441780
}

Submitted on 21 Oct 2020

HAL is a multi-disciplinary open access archive for the deposit and dissemination of scientific research documents, whether they are published or not. The documents may come from teaching and research institutions in France or abroad, or from public or private research centers.
L'archive ouverte pluridisciplinaire $\mathbf{H A L}$, est destinée au dépôt et à la diffusion de documents scientifiques de niveau recherche, publiés ou non, émanant des établissements d'enseignement et de recherche français ou étrangers, des laboratoires publics ou privés. 


\section{Ice-templated porous tungsten and tungsten carbide inspired by natural wood}

Yuan Zhang ${ }^{1,2}$, Guoqi Tan ${ }^{1,3}$, Da Jiao ${ }^{1}$, Jian Zhang ${ }^{1}$, Shaogang Wang ${ }^{1}$, Feng Liu ${ }^{2}$, Zengqian Liu ${ }^{1,3,}{ }^{*}$, Longchao Zhuo ${ }^{4, *}$, Zhefeng Zhang ${ }^{1,3,}$, Sylvain Deville ${ }^{5}$ \& Robert O. Ritchie ${ }^{6}$

${ }^{1}$ Laboratory of Fatigue and Fracture for Materials, Institute of Metal Research, Chinese Academy of Sciences, Shenyang 110016, China

${ }^{2}$ School of Mechanical Engineering, Liaoning Shihua University, Fushun 113001, China

${ }^{3}$ School of Materials Science and Engineering, University of Science and Technology of China, Hefei 230026, China

${ }^{4}$ School of Materials Science and Engineering, Xi'an University of Technology, Xi'an 710048, China

${ }^{5}$ Laboratoire de Synthèse et Fonctionnalisation des Céramiques, UMR3080 CNRS/Saint-Gobain CREE, Saint-Gobain Research Provence, Cavaillon 84306, France

${ }^{6}$ Department of Materials Science and Engineering, University of California Berkeley, Berkeley, CA 94720, USA

Abstract | The structures of tungsten and tungsten carbide scaffolds play a key role in determining the properties of their infiltrated composites for multifunctional applications. However, it is challenging to construct and control the architectures by means of self-assembly in W/WC systems because of their large densities. Here we present the development of unidirectionally porous architectures, with high porosities exceeding 65 vol.\%, for $\mathrm{W}$ and $\mathrm{WC}$ scaffolds which in many respects reproduce the design motif of natural wood using a direct ice-templating technique. This was achieved by adjusting the viscosities of suspensions to retard sedimentation during freezing. The processing, structural characteristics and mechanical properties of the resulting scaffolds were investigated with the correlations between them explored. Quantitative relationships were established to describe their strengths based on the mechanics of cellular solids by taking into account both inter- and intra-lamellar pores. The fracture mechanisms were also identified, especially in light of the porosity. This study extends the effectiveness of the ice-templating technique for systems with large densities or particle sizes. It further provides preforms for developing new nature-inspired multifunctional materials, as represented by $\mathrm{W} / \mathrm{WC}-\mathrm{Cu}$ composites.

Keywords: Ice-templating, Tungsten, Scaffolds, Fracture mechanisms, Bioinspired materials

\footnotetext{
* Corresponding authors.

Zengqian Liu (tel: +86-24-8397-0116, fax: +86-24-8397-0116, e-mail address: zengqianliu@imr.ac.cn); Longchao Zhuo (tel/fax: +86-29-8231-2516, e-mail address: zhuolongchao@xaut.edu.cn); Zhefeng Zhang (tel: +86-24-2397-1043, fax: +86-24-2389-1320, email address: zhfzhang@imr.ac.cn)
} 


\section{Introduction}

Tungsten (W) and tungsten carbide (WC) have ultrahigh melting points, high hardness, and outstanding resistance to wear and electrical erosion. These advantages can be combined with the high thermal and electrical conductivity of copper $(\mathrm{Cu})$ by forming W/WC-Cu composites [[1], [2], [3], [4], [5], [6]]. The excellent combinations of properties make the composites highly attractive for multifunctional applications. A common use, especially for the $\mathrm{W}-\mathrm{Cu}$ system, is to serve as high-voltage electrical contacts to resist the stringent arc erosion created by completing or interrupting the circuit, while simultaneously ensuring an efficient electrical conduction [[3], [4], [5], [6], [7], [8]]. Another good case in point is their potential as heat sinks for highly-loaded plasma facing components in nuclear fusion devices owing to their good thermal conductivity and superior mechanical properties at elevated temperatures [[9], [10], [11]]. WC-Cu composites are also promising candidates for thermal barriers between plasma facing components and copper-based heat sinks $[12,13]$. Other applications include the warhead and nozzle liners of missiles or rockets which utilize high densities and the unique thermophysical-mechanical properties of the composites $[14,15]$.

A viable approach to fabricate $\mathrm{W} / \mathrm{WC}-\mathrm{Cu}$ composites is to infiltrate a $\mathrm{Cu}$ melt into the open pores of W/WC scaffolds [[1], [2], [3], [4], [5], [6]]. This is made possible by the large gap in the melting temperatures of W/WC and $\mathrm{Cu}$ (the melting points are $3422^{\circ} \mathrm{C}$ and $2870{ }^{\circ} \mathrm{C}$ for $\mathrm{W}$ and $\mathrm{WC}$, respectively, but is only $1083^{\circ} \mathrm{C}$ for $\mathrm{Cu}$ ) and the minimal mutual solubility or interfacial reaction between them $[14,16]$. In this scenario, the structure of W/WC scaffolds can be eventually inherited by the composites which can play a key role in dictating their final properties. These scaffolds are most commonly processed with powder metallurgy techniques because of the refractory nature of $\mathrm{W}$ and WC [[1], [2], [3], [4], [5], [6], [7], [8], [9], [10], [11], [12], [13], [14], [15]]. Despite its good applicability, such a method encompasses several shortcomings that may downgrade the performance of resulting composites. Firstly, the scaffolds generally contain a limited fraction of pores, with porosity typically smaller than $50 \mathrm{vol} \%$ [ [ 1$]$, [2], [3], [4], [5], [6], [7], [8], [9], [10], [11], [12], [13], [14], [15]], to maintain their integrity during processing. This leads to a low $\mathrm{Cu}$ content in the infiltrated composites and accordingly restricts their thermal and electrical conductivity. Secondly, the pores in the scaffolds are essentially isometric in geometry and randomly distributed. This makes the conduits for thermal and electrical transport rather narrow and torturous along any direction in the composites as they are primarily localized in the $\mathrm{Cu}$ component. Such an isotropic protocol for processing these materials contrasts sharply with their property requirements which are directional in most practical service conditions [[2], [3], [4], [5], [6], [7], [8], [9], [10], [11], [12], [13]]; indeed, this is especially true for thermal and electrical conduction properties which are the major function of W/WC-Cu composites.

Differing markedly from man-made materials where the properties are derived largely from chemical complexity, natural materials are particularly adept at developing their 
functionalities based on the optimization of architectures [[17], [18], [19], [20]]. Wood is an excellent example of multifunctional scaffolds in nature [[21], [22], [23], [24], [25], [26]]. It features a porous structure, comprising the wood cells, vessels, tracheids, and sieve tubes, which is highly oriented along the growth direction of plant stems. This maximizes the axial stiffness and strength for robust mechanical support, and simultaneously enables an effective transport of water, salts and assimilates in an "upstem-downstem" fashion. Additionally, wood exhibits limited heat transfer in the transverse profile, endowed by its longitudinal pores; as such, wood can function as a good heat insulator for some plant species [25,26], which in certain respects is analogous of the application of W/WC-Cu composites as thermal barriers. Such architecture makes the wood an idealized natural prototype for man-made porous materials. Specifically, it may provide inspiration for new W/WC scaffolds for subsequent melt infiltration, thus offering the promise of enhanced multi-functionality in $\mathrm{W} / \mathrm{WC}-\mathrm{Cu}$ composites.

In this study, highly-porous W and WC scaffolds with unidirectionally-oriented architectures, which in many respects mimic wood, were fabricated based on a direct ice-templating (freeze casting) technique. W/WC powders were suspended in water and assembled into ordered lamellae by freezing. The microstructure, mechanical properties and fracture mechanisms of the scaffolds were systematically explored and correlated to the initial solid loading of suspensions. We believe these scaffolds can promote the development of new multifunctional W/WC-Cu composite materials.

\section{Experimental}

\subsection{Processing}

Stable aqueous-based suspensions were prepared for ice-templating by dispersing submicrometer-sized W and WC powders (Pingyuan Alloy Co., China) with average diameters of $\sim 500 \mathrm{~nm}$ and $300 \mathrm{~nm}$, respectively, in deionized water. The powders were made electrostatically repulsive by surface charging with anions with the addition of 0.5 wt.\% (relative to the powders) Darvan CN dispersant (R.T. Vanderbilt Co., CT). The viscosities of slurries were adjusted by adding different amounts of hydroxypropyl methylcellulose (HPMC, Meryer Co., China). The formation of hydrogen bonds between HPMC and water molecules functions to increase the flow resistance of slurries [27]. The amounts of HPMC additives to suspend $\mathrm{W}$ and $\mathrm{WC}$ powders were determined to be 5.5 and $2.5 \mathrm{wt} . \%$, respectively, with respect to water, in view of their different densities (the densities of $\mathrm{W}$ and $\mathrm{WC}$ are 19.35 and $15.63 \mathrm{~g} / \mathrm{cm}^{3}$, respectively). Additionally, 1 wt.\% poly(vinyl alcohol) (molecular weight of 84-89 kDa, Meryer Co., China) and $1 \mathrm{wt} . \%$ sucrose were added to bind the powders after removal of the water. Specifically, nano-sized $\mathrm{Cu}$ powders $(\sim 50 \mathrm{~nm}$ in diameter, Huisheng New Materials Co., China) accounting for $3 \mathrm{wt} . \%$ of solids were added as a sintering aid for the $\mathrm{W}$ system. The contents of solid powders with respect to the total of solids and water were $75,80,85$, and $90 \mathrm{wt} . \%$ for $\mathrm{W}$ suspensions and 55, 65, 75, and $85 \mathrm{wt} . \%$ for WC. 
Accordingly, by taking the additives into account the total solid loads were 71.3, 76.3, 81.3, and 86.4 wt.\% for W and 53.7, 63.8, 73.9, 84.1 wt.\% for WC systems. Slurries of the same solid loads but without HPMC additions were also prepared for comparison.

The mixtures were ball-milled for $48 \mathrm{~h}$ and de-aired with drops of defoamer (XP-M120, Huaxing Co, China) before use. Bidirectional ice-templating was performed by pouring the slurries into square plastic molds in dimensions of $20 \mathrm{~mm} \times$ $20 \mathrm{~mm} \times 70 \mathrm{~mm}$. The bottoms of the molds were plugged using polydimethylsiloxane (PDMS) wedges with a slope angle of $25^{\circ}$ [28]. The molds were cooled from the bottom to up by placing them on a copper plate where the other end was immersed in liquid nitrogen. Adequate freezing of slurries, with height of $\sim 40 \mathrm{~mm}$, was accomplished in $\sim 1 \mathrm{~h}$, giving an average freezing front velocity of $\sim 10 \mu \mathrm{m} / \mathrm{s}$. The frozen samples were removed from their molds and then freeze-dried in a vacuum lower than $5 \mathrm{~Pa}$ for over $64 \mathrm{~h}$. The organics in the samples were removed by heat treatment at $800^{\circ} \mathrm{C}$ for $5 \mathrm{~h}$ in flowing argon gas. The $\mathrm{W}$ and $\mathrm{WC}$ scaffolds were sintered at $1350^{\circ}$ and $1800^{\circ} \mathrm{C}$, respectively, for $2 \mathrm{~h}$ in vacuo. A lower sintering temperature was chosen for $\mathrm{W}$ than WC despite its higher melting point because of the use of $\mathrm{Cu}$ nano-powders as sintering aid. Specifically, the $\mathrm{W}$ samples were heat treated below $900{ }^{\circ} \mathrm{C}$ for $5 \mathrm{~h}$ before sintering in a flowing atmosphere of 95 vol. $\%$ argon and 5 vol. $\%$ hydrogen to eliminate possible oxidation. The infiltration of $\mathrm{W}$ scaffolds with a $\mathrm{Cu}$ melt was conducted at $1350{ }^{\circ} \mathrm{C}$ for $1 \mathrm{~h}$ in flowing argon gas.

\subsection{Characterization}

The viscosities of the slurries were measured using a NDJ-8S digital rotary viscometer (Decca Precision Instrument Co., China). The nominal densities of sintered scaffolds were determined using the Archimedes method. Microstructural characterization was performed on the top surfaces of freeze-dried green bodies and on the longitudinal and cross-sectional profiles of sintered scaffolds for both W and WC systems. The samples were extracted from the middle regions of the scaffolds to exclude the influence of possible structural heterogeneity along the height direction caused by the difference in freezing front velocity [[28], [29], [30]]. The green bodies were examined using an Olympus LEXT OLS-4000 confocal laser microscope. The scaffolds were characterized by scanning electron microscopy (SEM) using a LEO Supra 55 field emission microscope operating at an accelerating voltage of $20 \mathrm{kV}$. The SEM images were analyzed using the Image-Pro software (Meyer Instruments, Inc., TX) to determine the microstructural characteristics, such as the thickness and spacing of lamellae, the bridges and intersections between them, and the dimensions and geometries of the pores. Over 100 lamellae, with a measured area over $5 \mathrm{~mm}^{2}$, were examined for each sample in order to quantify the parameters. The three-dimensional morphologies of the scaffolds, and representative woods of $O$. pyramidale (balsa wood) and B. schmidtii (birch wood) with markedly differing densities, were scanned and reconstructed using an Xradia VersaXRM-500 3D X-ray tomography (XRT) system operating at an accelerating voltage of $80 \mathrm{kV}$. The image processing and analysis were conducted using the Avizo Fire 7.1 software. 
Rectangular samples, with dimensions of $5 \mathrm{~mm} \times 5 \mathrm{~mm} \times 10 \mathrm{~mm}$, were machined from the scaffolds using a precision diamond wire saw (STX-202A, Kejing Auto-Instrument Co., China) equipped with a diamond-coated stainless wire with a diameter of $0.3 \mathrm{~mm}$. The height direction of samples coincided with the long axis of scaffolds, i.e., the growth direction of ice during freezing. A low cutting speed of $0.1 \mathrm{~mm} / \mathrm{min}$ was employed to minimize the damage introduced by the cutting process. Uniaxial compression tests were performed on the samples at room temperature using an Instron E-1000 testing system with a fixed strain rate of $10^{-3} \mathrm{~s}^{-1}$. At least four samples were tested for each set of scaffolds. After testing, SEM imaging was performed to characterize the fracture mechanisms.

\section{Results}

\subsection{Rheological properties of suspensions}

The viscosities of the suspensions were adjusted in order to prevent the gravitational sedimentation of $\mathrm{W}$ and $\mathrm{WC}$ powders during the freezing process because of their large densities. This was realized by adding HPMC, a water-soluble methylcellulose ether containing methoxy and hydroxypropyl groups [27], as a thickening agent. The viscosities of the aqueous solutions are markedly enhanced (Fig. 1(a)), by some six orders of magnitude (the viscosity of water is around $10^{-3} \mathrm{~Pa} \cdot \mathrm{s}$ ), with the addition of $3 \mathrm{wt}$ \% HPMC (the viscosities for solutions containing more HPMC are unavailable here as they can hardly flow). Such trends can be fitted with an exponential-type relationship as [31,32]:

$\eta=A e^{-w / B}+C$

where $\eta$ and $w$ are, respectively, the viscosity and the additive amount by weight, with, and $\mathrm{A}, \mathrm{B}$ and $\mathrm{C}$ as fitting parameters. The viscosities of final suspensions for freezing vary in similar fashion as a function of the solid loads of W and WC (Fig. 1(b), (c)). Nevertheless, they demonstrate a much stronger dependence on the content of HPMC as compared to the solids loads (as shown by the results in the Supplementary Materials), indicating the potency of HPMC in thickening the slurries. 

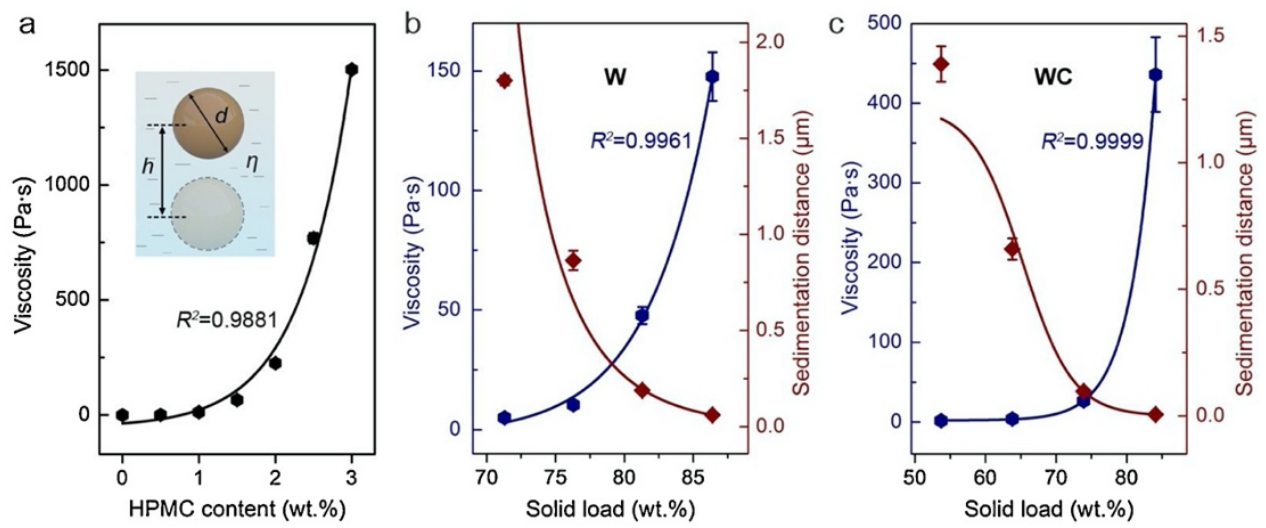

Fig. 1. (a) Viscosities of aqueous solutions containing varying contents of HPMC. The inset illustrates the sedimentation of a solid powder with diameter $d$ in a viscous liquid with viscosity $\eta$ caused by gravity. (b, c) Variations in the viscosities of suspensions and the sedimentation distances of solid powders during the freezing process as a function of the solid load for the (b) $W$ and (c) WC systems. The fitting curves for viscosities were obtained from Eq. (1) with the goodness-of-fit indicated in the figure. The sedimentation distances were fitted according to Eq. (S2) in the Supplementary Materials.

The significantly increased viscosities of suspensions play an effective role in retarding the sedimentation of solid powders caused by gravity, as illustrated in the inset in Fig. 1(a). The terminal velocities for steady-state sedimentation can be obtained, from Stokes' law [33], to be less than $2 \mu \mathrm{m} / \mathrm{h}$ for the $\mathrm{W}$ and WC systems (detailed derivations are provided in the Supplementary Materials). With respect to the actual case where the powders are originally stationary, the sedimentation distance can be accessed by integrating the forces of gravity, buoyancy, and the viscous friction by Stokes' drag. The maximum sedimentation distances were determined to be less than $2 \mu \mathrm{m}$, i.e., only slightly larger than the diameters of powders, over the entire freezing process of $\sim 1 \mathrm{~h}$ for all the W and WC suspensions (Fig. 1(b), (c)). By contrast, the slurries without HPMC additions exhibit obvious sedimentation within minutes with the powders concentrated towards the bottom (Fig. S2 in Supplementary Materials). Such effective stabilization of the suspensions makes it possible to assemble the powders into aligned lamellae by ice-templating.

\subsection{Microstructure}

\subsubsection{Unidirectionally porous architectures}

The top-surface morphologies of the freeze-dried $\mathrm{W}$ and $\mathrm{WC}$ green bodies reveal the presence of elongated pores on the transverse cross-sections (Fig. S3 in Supplementary Materials). The structural characteristics differ between the two systems and vary with the solid loads. Specifically, the struts exhibit decreased ordering and uniformity in thickness as they become highly intersected with each other as the solid load increases 
for the W green bodies. By comparison, all the WC green bodies have well-defined lamellae and preferentially aligned pores, but display apparent intersection of struts at a high solid load of 84.1 wt.\%.

The main structural features of green bodies are inherited into the scaffolds after sintering. As shown in Fig. 2, the longitudinal profiles of both W and WC scaffolds demonstrate unidirectional pores along the growth direction of the ice during the freezing process. There is a global trend of decreasing porosity, increasing lamellar wall thickness, and increasing interconnectivity between lamellae in the form of bridging or bifurcation with the increase in solid load. The three-dimensional and cross-sectional morphologies of the scaffolds are shown in Fig. 3 and compared with those of representative woods with differing densities (Fig. S4 in Supplementary Materials). The W/WC scaffolds are similar to natural wood in their unidirectionally porous architectures, despite their markedly different constituents and characteristic dimensions. In many respects, this represents an implementation of the material-design principle of wood rather than a rigid replication of its structure. Although there are much more complex designs at differing length-scales in wood, the unidirectionally porous architecture offers a prime source for its unique multi-functionality [[21], [22], [23], [24], [25], [26]]. Specifically, the WC scaffolds display more directional alignment of lamellae than the $\mathrm{W}$ system, particularly over the transverse profile.
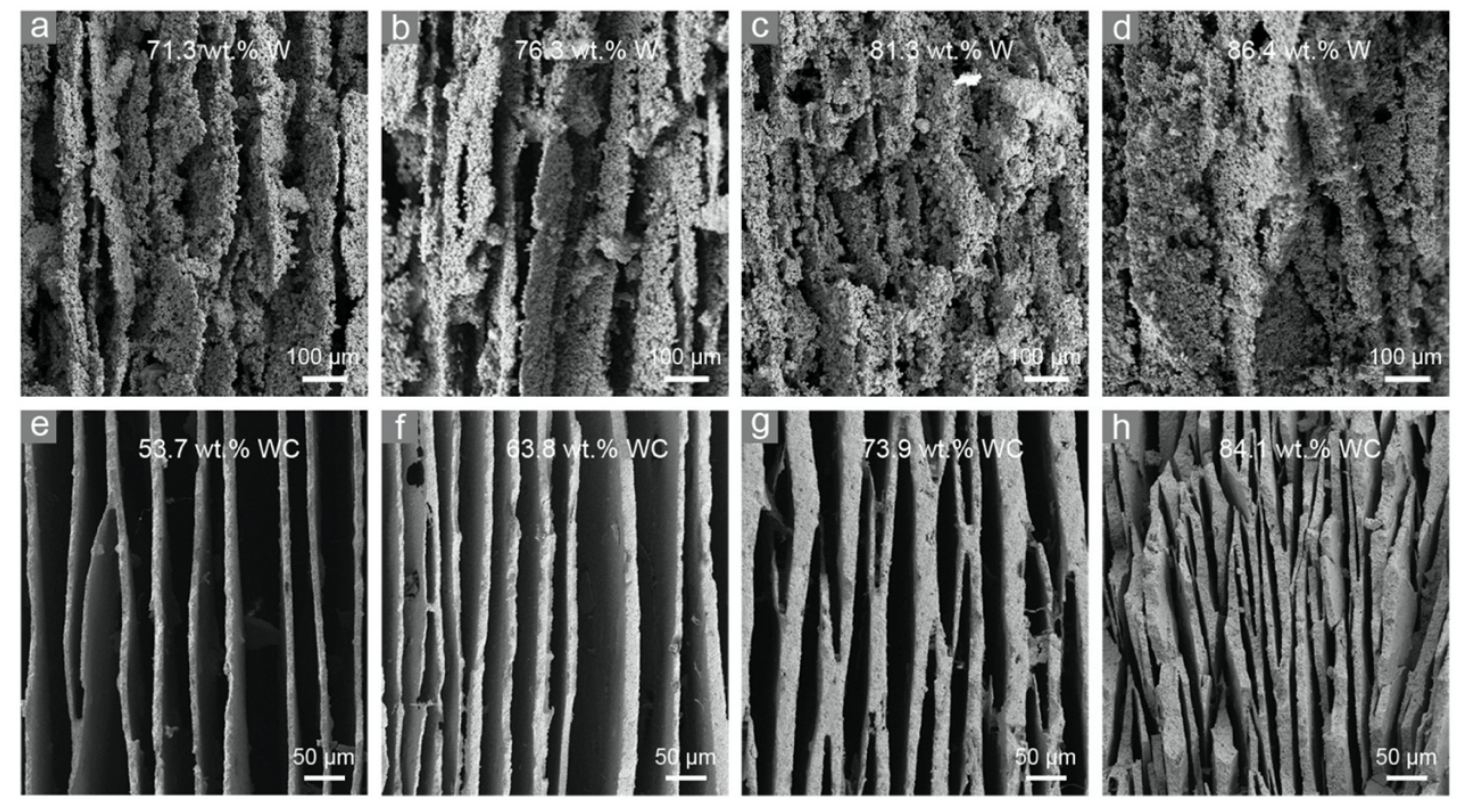

Fig. 2. SEM morphologies of the longitudinal cross-sections at the middle regions of sintered scaffolds with differing solid loads for $(a-d) W$ and $(e-h) W C$ systems. 

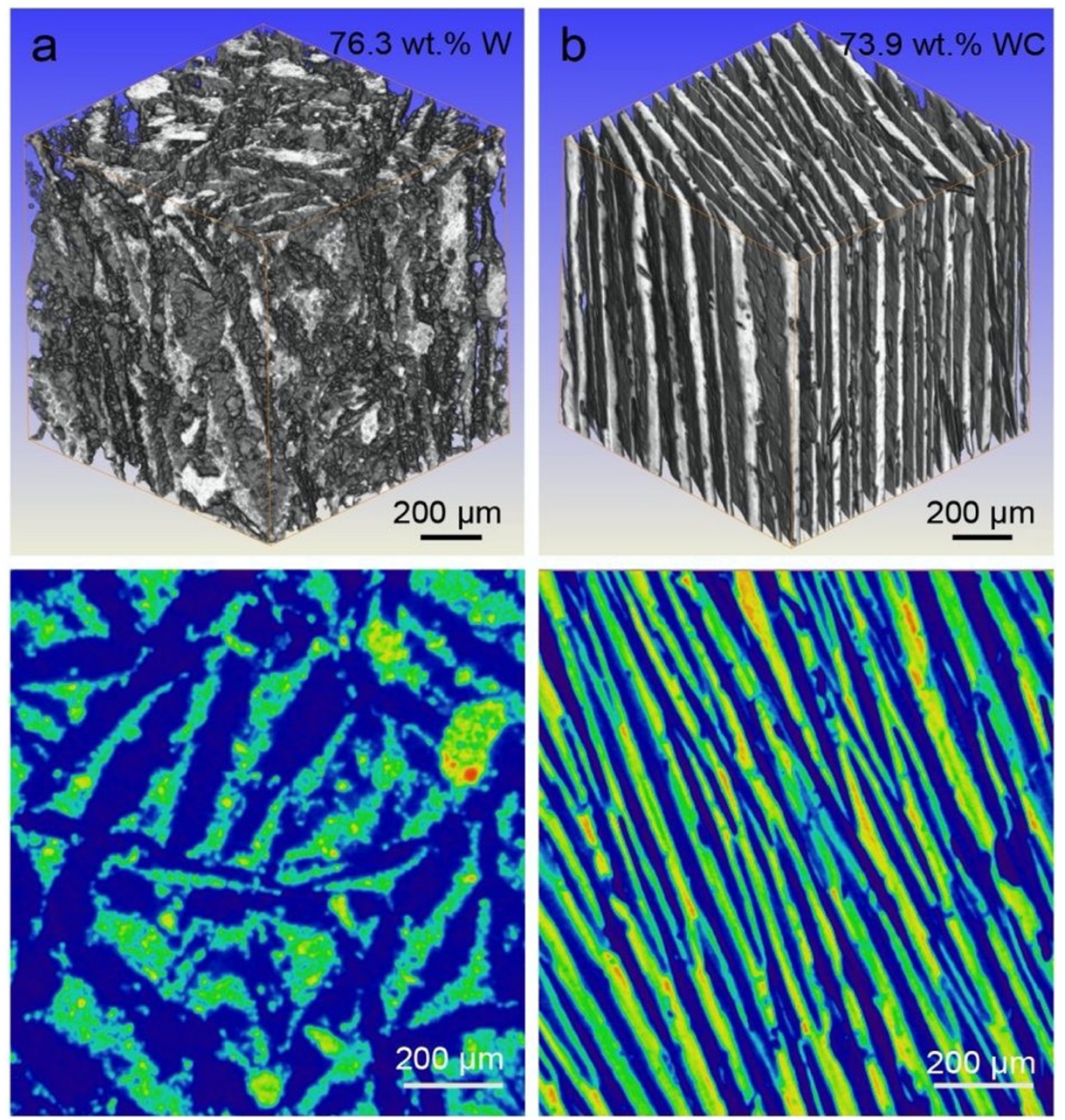

Fig. 3. Three-dimensional XRT volume renderings and corresponding cross-sectional slices of sintered scaffolds of the (a) W and (b) WC systems with 76.3 and 73.9 wt.\% solid loads, respectively.

\subsubsection{Structural characteristics}

Fig. 4 presents schematic illustrations of the structural characteristics of scaffolds for quantitative description. There are two types of pores in the scaffolds - the unidirectional pores between lamellae and the smaller pores involved within lamellae, referred to in the following as inter- and intra-lamellar pores. Here the total volume fraction of porosity, $P_{\text {total }}$, in the scaffolds was determined from the nominal density, $\rho$, according to $P_{\text {total }}=1-\rho / \rho_{S}$ with $\rho_{S}$ as the density of dense W or WC. The inter-lamellar porosity was measured by image analysis with the intra-lamellar porosity calculated by subtracting inter-lamellar porosity from the total. 


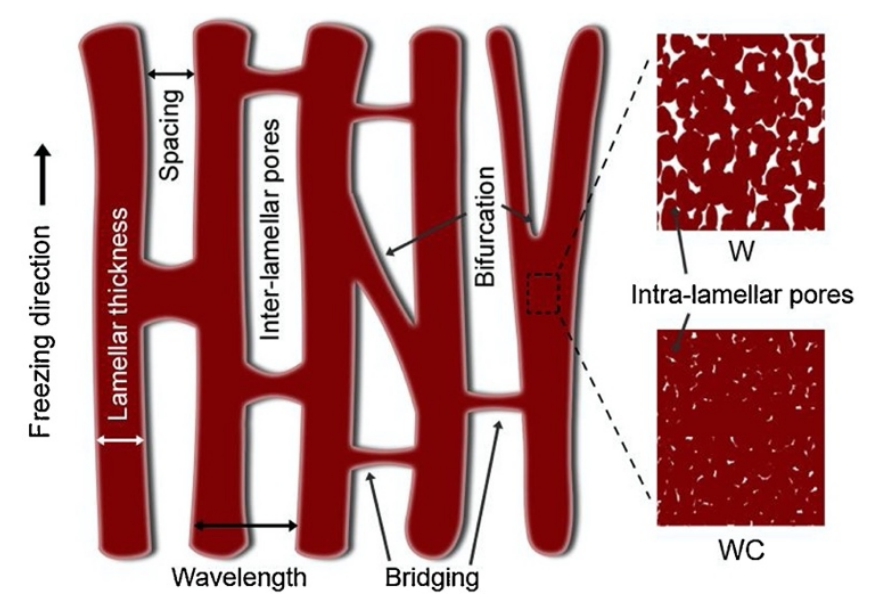

Fig. 4. Schematic illustrations of the structural characteristics of the $W$ and $W C$ scaffolds and magnified views of their lamellae indicating the intra-lamellar pores.

The lamellar thickness increases with increasing solid load (Fig. 5(a)), specifically in a steeper fashion for the $\mathrm{W}$ system, with average values ranging from 50 to $140 \mu \mathrm{m}$ in $\mathrm{W}$ scaffolds, but less than $35 \mu \mathrm{m}$ for WC. All the scaffolds exhibit large total porosities exceeding 65 vol.\% which are negatively correlated with the solid load (Fig. 5(b)). The intra-lamellar porosities increase monotonically with increasing solid load, and account for a large proportion, up to $\sim 45 \mathrm{vol} . \%$, for the $\mathrm{W}$ system. The inter-lamellar porosities show an opposite varying trend yet still contribute the majority of the total porosity in the WC scaffolds. The differing constitutions of porosity between $\mathrm{W}$ and WC systems are directly associated with the degree of densification in their lamellae. The powders are loosely packed and poorly interconnected within the lamellae of $\mathrm{W}$ scaffolds (Fig. 5(c)), which contrasts to the dense sintering of WC. Additionally, the aspect ratios of inter-lamellar pores display a decreasing trend as the solid load increases, and are relatively larger for the WC system (Fig. 5(d)). 

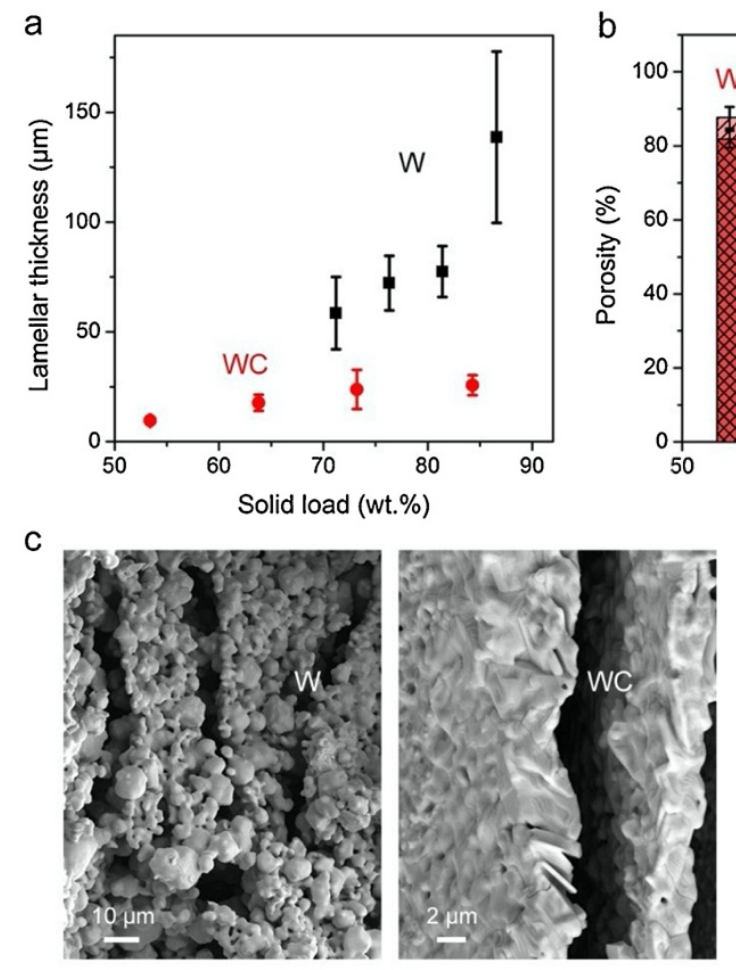

b
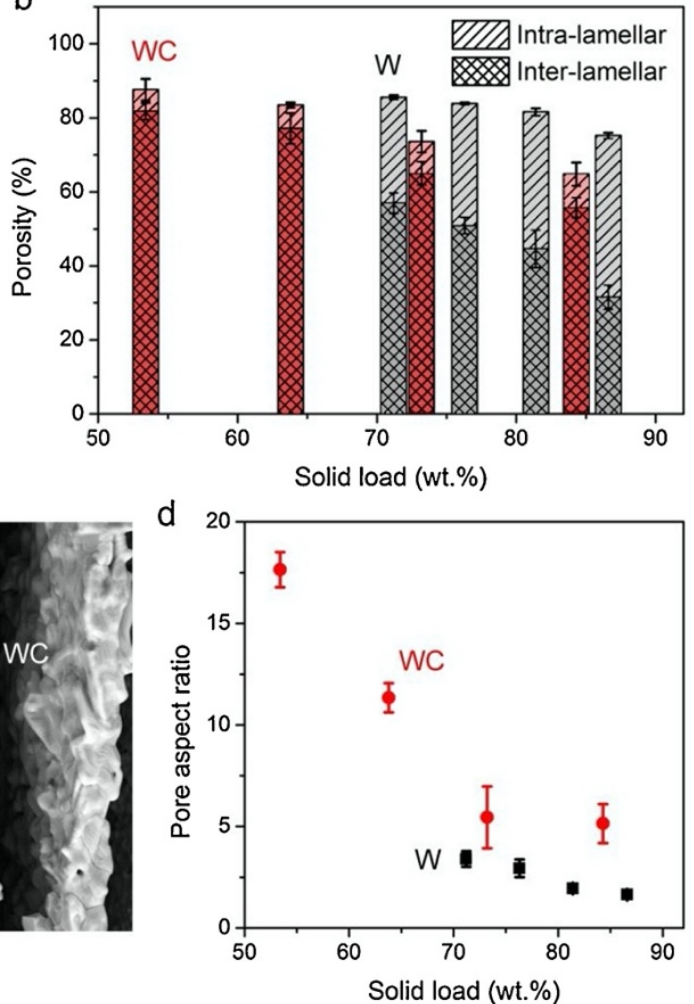

Fig. 5. Structural characteristics of the $W$ and WC scaffolds. (a,b) Variations in the (a) lamellar thickness and (b) inter-and intra-lamellar porosities of the $W$ and WC scaffolds as a function of solid load. The data are expressed in the form of average \pm standard deviation. Vertical columns with their centers along the $\mathrm{x}$-axis denoting specific solid loads are used in (b) for clarity. (c) SEM images of the lamellae showing differing degrees of densification between the W and WC systems. (d) Dependences of the aspect ratios of inter-lamellar pores on the solid loads.

The interconnections between lamellae can be categorized into two different types: bridging that is nearly perpendicular to the lamellae, and bifurcation with an inclination angle typically lower than $45^{\circ}$ with respect to the lamellae (inset in Fig. 6(a)). Specifically, an interconnection is seen as a pair of bifurcations when its width exceeds the wavelength of the lamellae $\lambda$, as illustrated in Fig. 6(b). Bridging and bifurcation were found to dominate the interconnectivity in the $\mathrm{W}$ and $\mathrm{WC}$ scaffolds, respectively, and show increasing densities in both systems as the solid load increases. 

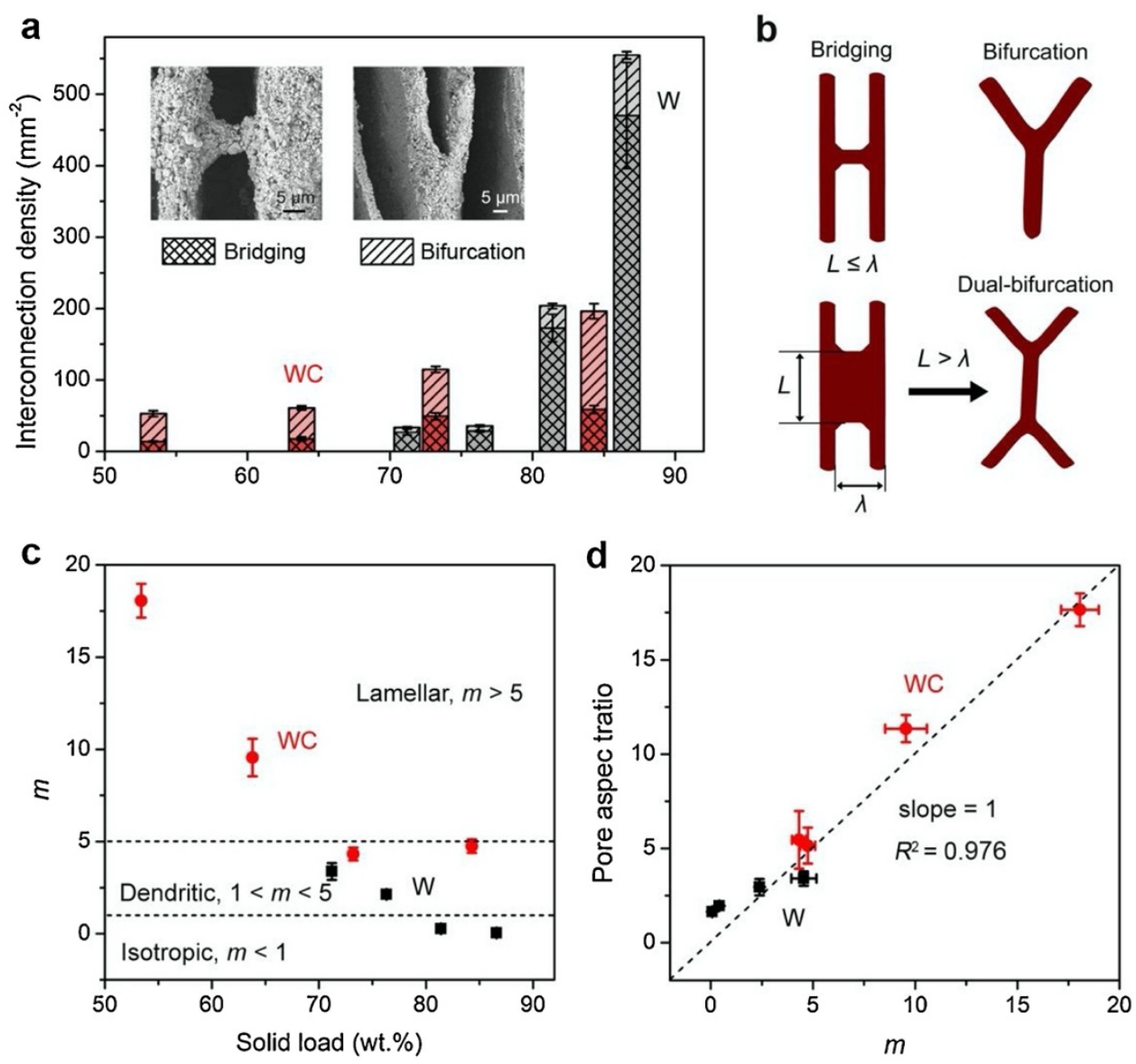

Fig. 6. (a) Variations in the area densities of the interconnections between lamellae as a function of the solid load for $W$ and $W C$ scaffolds. The insets show representative morphologies of lamellar bridging and bifurcation. (b) Schematic illustrations of the interconnections between lamellae for quantitative analysis. (c) Variations in the parameter $m$ and the structural morphologies of the $W$ and WC scaffolds with solid load. (d) Relationship between the parameter $m$ and the aspect ratios of inter-lamellar pores for the scaffolds.

A dimensionless parameter $m$ has been proposed by Naglieri et al. to describe the morphology of ice-templated scaffolds [34,35], viz.:

$m=1 /\left(\rho_{i} \lambda^{2}\right)$

where $\rho_{i}$ is the area density of interconnections between lamellae. The differing structures with lamellar, dendritic or isotropic morphologies can be distinguished by comparing the spacing between interconnections and the wavelength of lamellae. The scaffold is considered as lamellar type for $m>5$ where the spacing is markedly larger than the wavelength. A dendritic structure is identified when the spacing and wavelength are comparable in terms of $1<m<5$. For $m<1$, there are dense interconnections with spacing smaller than the wavelength, leading to nearly isotropic structure. Here the normalized densities of interconnections were obtained by treating a bifurcation as one-half of bridging with $\rho_{i}=\rho_{\text {bridging }}+0.5 \rho_{\text {bifurcation }}$. As shown 
in Fig. 6c, the parameter $m$ demonstrates a general decreasing trend as the solid load increases. This leads to structural transitions of dendritic-to-isotropic for the $\mathrm{W}$ system and lamellar-to-dendritic for the WC system. Additionally, the aspect ratios of interlamellar pores, specifically the ratios of their length to width (Fig. 4), present a good correspondence with the parameter $m$ (Fig. 6d), indicating its pertinence as a descriptor of these structures.

\subsection{Mechanical properties}

The representative compressive stress-strain curves (Figs. 7(a), 7(b)) demonstrate markedly improved strengths of W and WC scaffolds with an increase in solid load, i.e., due to the decrease in total porosity. The $\mathrm{W}$ scaffolds exhibit varying degrees of nominal plasticity which decreases with increasing solid load (i.e., decreasing porosity); these scaffolds fracture by collapsing into tiny fragments, as shown in the inset. By contrast, the WC system displays a fracture mode of splitting into large pieces of lamellae with virtually no evidence of plasticity. The compressive strengths range from $0.65 \pm 0.07$ to $4.3 \pm 0.5 \mathrm{MPa}$ for the $\mathrm{W}$ scaffolds and from $0.81 \pm 0.09$ to $15 \pm 3 \mathrm{MPa}$ for WC scaffolds (Fig. 7(c), 7(d)). The relatively lower strengths of the present $\mathrm{W}$ scaffolds, specifically as compared to those made by the reduction of sintered $\mathrm{WO}_{3}$ [36], may be associated with the loose packing of $\mathrm{W}$ powders within the lamellae (Fig. 5(c)). Additionally, the energy absorption density of these scaffolds, denoted in terms of the area under stress-strain curves up to the maximum stress before fracture, displays an increasing trend as the solid load increases in both systems.

The fracture morphologies reveal the preferred damage initiation and collapse of $\mathrm{W}$ scaffolds at the contact area during compression tests (Fig. 8(a), 8(b)). The lamellae are microscopically plastic, which is apparent from their bending deformation (Fig. 8(b)) due to the metallic nature of $\mathrm{W}$. The existence of minimal $\mathrm{Cu}$ in the lamellae, which was introduced as a sintering aid and displays little mutual solubility with $\mathrm{W}$, can play an additional role in promoting micro-plasticity. The progressive collapse by separation between grains within the lamellae leads to a zig-zag fracture profile in the scaffolds. Despite this process, the scaffolds are still capable of bearing compressive loads and thereby exhibit certain nominal plasticity at the macroscale. In comparison, the WC scaffolds tend to undergo brittle rupture with minimal plasticity because of their ceramic nature (Fig. 8(c), (d)). The densely sintered lamellae tend to display a high fracture resistance, specifically as compared to the breakage of interconnections between them. This results in the preferred separation between lamellae, causing the splitting fracture of scaffolds. 

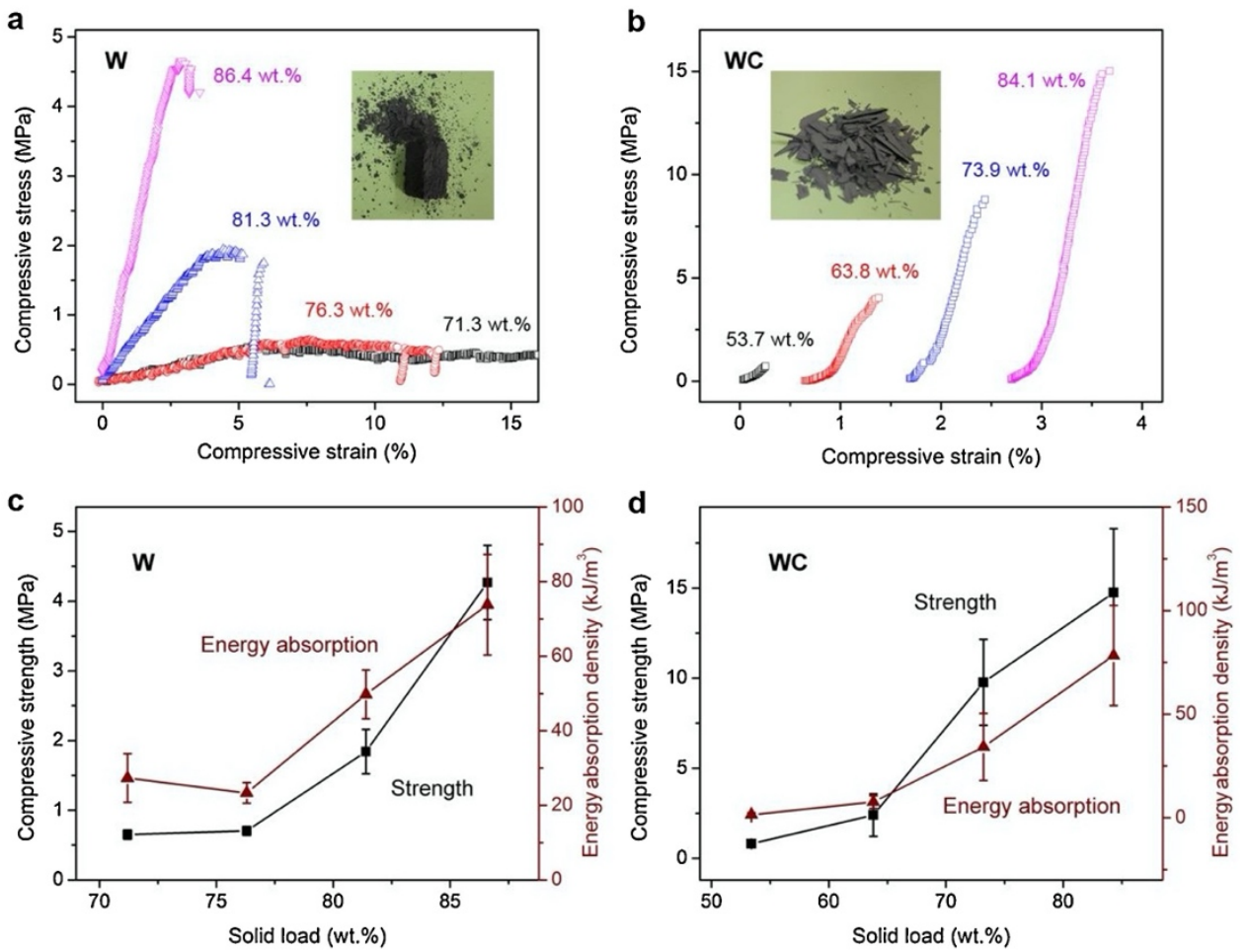

Fig. 7. Mechanical properties of the $W$ and $W C$ scaffolds. (a, b) Representative compressive stress-strain curves of the (a) $W$ and (b) WC scaffolds with varying solid loads. The curves are shifted horizontally for clarity. The insets show typical macroscopic morphologies of the scaffolds after failure. (c, d) Variations in the (c) compressive strengths and (d) energy absorption densities before failure of the scaffolds as a function of the solid load. 

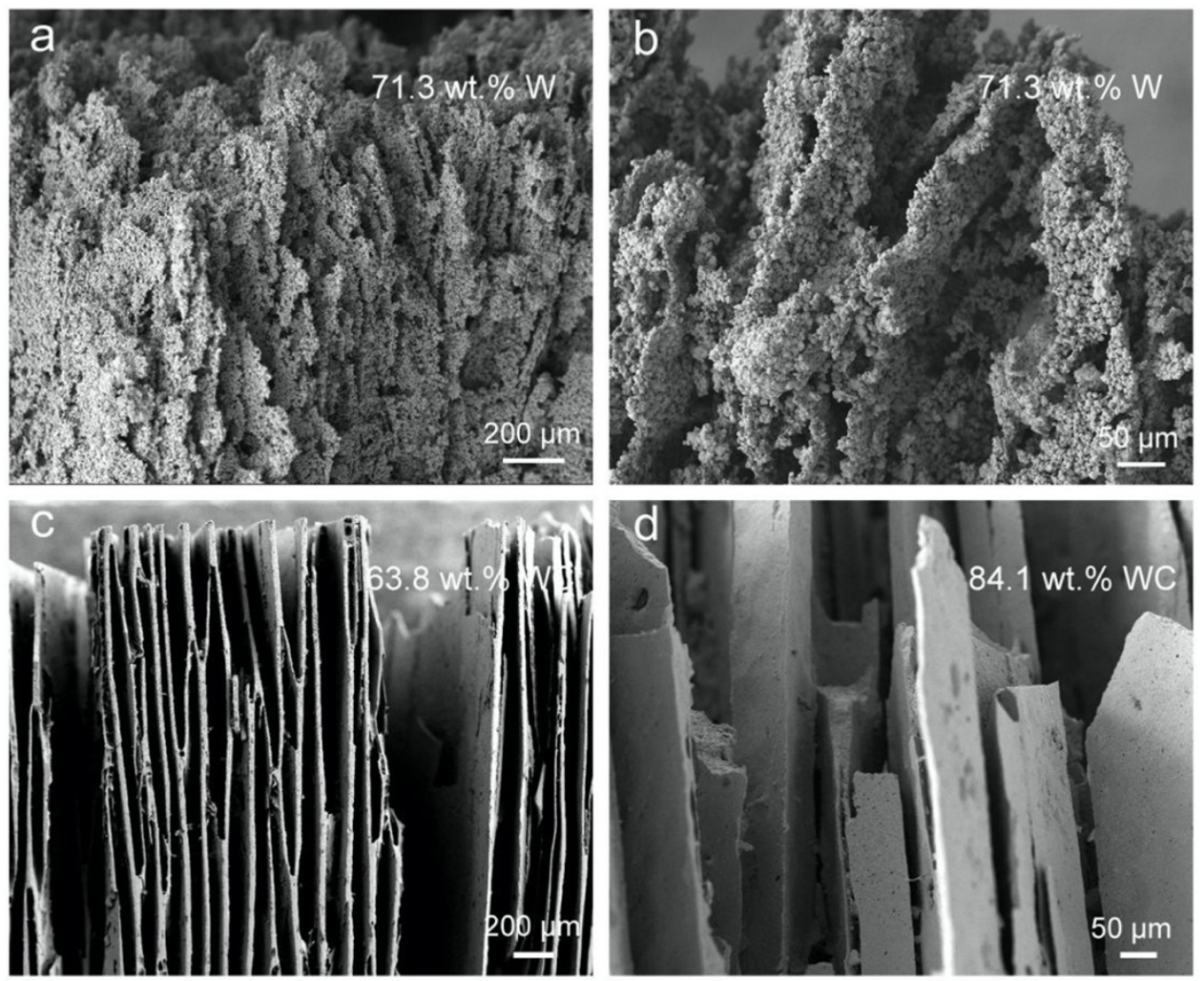

Fig. 8. Typical SEM morphologies of fractured samples for the (a,b) W and (c, d) WC scaffolds after compression testing.

\section{Discussion}

\subsection{Processing}

Ice-templating, also known as freeze casting, has been widely used in fabricating porous polymer, ceramic and metal scaffolds by providing an effective means to create unidirectional micro-pores [29,30,34,[37], [38], [39], [40], [41], [42], [43], [44], [45]]. During the directional freezing process of a suspension, the growing ice crystals repel and concentrate the particles and additives between adjacent growing crystals. This allows for the construction of lamellar architectures in the scaffolds after the sublimation of the ice. However, the ice-templating of $\mathrm{W}$ and $\mathrm{WC}$ systems is challenging from the perspective of making stable suspensions because their densities are so high, respectively, 19.35 and $15.63 \mathrm{~g} / \mathrm{cm}^{3}$. The large settling tendency of heavy powders in the slurry can lead to a marked inhomogeneity of the scaffolds and even prevent the formation of ice lamellae $[46,47]$. Specifically, destabilization of the solidliquid interface during freezing caused by flocculation may trigger the formation of an ice lens and create structural defects in the lamellae [47].

Rothlisberger et al. [36,48] and Lee et al. [49] have developed unidirectional W foams by reducing ice-templated tungsten oxide $\left(\mathrm{WO}_{3}\right)$ which has a much lower density $\left(7.27 \mathrm{~g} / \mathrm{cm}^{3}\right)$ than W and WC. Similar methods have also been employed in fabricating cobalt $(\mathrm{Co})$ and iron $(\mathrm{Fe})$ foams using cobaltosic oxide $\left(\mathrm{Co}_{3} \mathrm{O}_{4}\right)$ and hematite $\left(\mathrm{Fe}_{2} \mathrm{O}_{3}\right)$ 
as preforms [50,51]. However, such strategies may have several limitations compared to the direct templating of final products. They are restricted to materials which form an oxide that can be reduced back; nevertheless, these materials per se need to be inactive to hydrogen or other reducing agents. Additionally, the complete reduction of oxides often necessitates a long treatment time and considerable energy input. Here, the sedimentation of powders is retarded by improving the viscosities of suspensions with appropriate additives, particularly using HPMC as a thickener. Adjustments in viscosity have also been shown to be effective in suspending large alumina platelets in aqueous slurries [52]. By such means, the feasibility of ice-templating technique can be enhanced to a broader range of material-systems, thereby allowing for the easy fabrication of unidirectional scaffolds, specifically for solids having large densities or particle sizes.

On the other hand, an increase in viscosity may retard the repulsion of W/WC particles from the growing ice front during the freezing process. This tends to increase the propensity of particle engulfment which may lead to disordered porous structures and irregular shapes of pores $[46,47,53]$. The engulfed particles may also form bifurcations or bridges after subsequent sintering, thereby enhancing the interconnectivity between lamellae. Such engulfment is believed to be promoted by HPMC additions in the present W/WC systems, but does not impede the formation of unidirectionally porous structures in the final scaffolds. Additionally, there is an upper limit of porosity in the scaffolds made by ice-templating technique because the solid load in suspension cannot be too low so as to maintain the integrity of green bodies. Also, an inferior-limit of porosity also exists to avoid the sedimentation of solid powders in suspension while keeping satisfied flowability. A wide range of porosity from lower than $20 \mathrm{vol} . \%$ to around 90 vol.\% has been reported for ice-templated ceramic scaffolds [29,39,40,43]. The exact values of such limits have not been experimentally accessed in current study.

The differences in the porous morphologies between $\mathrm{W}$ and $\mathrm{WC}$ scaffolds are mainly caused by the differing solid loads and viscosities of suspensions for freeze casting along with their distinctly different sintering temperatures. Specifically, the larger tendency of sedimentation for $\mathrm{W}$ powders due to its higher density than WC leads to decreased ordering of lamellar structure in freeze-cast scaffolds [46,47]. Additionally, the sintering temperature of $\mathrm{W}$ scaffolds is only $\sim 0.39$ times of its melting point owing to the use of sintering aid (by comparison, the value is $\sim 0.63$ for the sintering of WC scaffolds). This is responsible for the relatively lower degree of densification in the lamellae of W scaffolds.

\subsection{Strength}

The strength of a cellular solid is determined by its relative density or porosity with a dependence associated with the architectural arrangement. Based on the mechanics of the Gibson-Ashby model [54], the strength of an open-cell foam with random pores varies in power-law fashion as a function of the porosity $P$ as: 
$\sigma / \sigma_{s} \propto(1-P)^{1.5}$

where denotes the strength of a fully dense solid. In the case of unidirectional pores, as represented by honeycomb structures, the out-of-plane strength of the foam scales linearly with its relative density in the form as [54]:

$\sigma / \sigma_{s} \propto(1-P)$.

The strength of the present $\mathrm{W}$ and WC scaffolds cannot be captured by either of the above relationships (Fig. 9(a)). This is probably because the inter- and intra-lamellar pores are, respectively, aligned and randomly organized. Their effects can be integrated by assuming a unidirectional foam comprising walls of an isotropic cellular material (inset in Fig. 9(b)). In this scenario, the strength of the lamellar wall, , $\sigma_{l}$, can be described by the following derivation:

$\sigma_{l} / \sigma_{S} \propto\left[\left(1-P_{\text {total }}\right) /\left(1-P_{\text {inter }}\right)\right]^{1.5}$

where and are, respectively, the total and inter-lamellar porosities. The term within the square brackets indicates the relative density of lamellae; as such, the strength of the entire foam can be associated with that of the wall by taking the unidirectional pores into account as:

$\sigma / \sigma_{l} \propto\left(1-P_{\text {inter }}\right)$ 

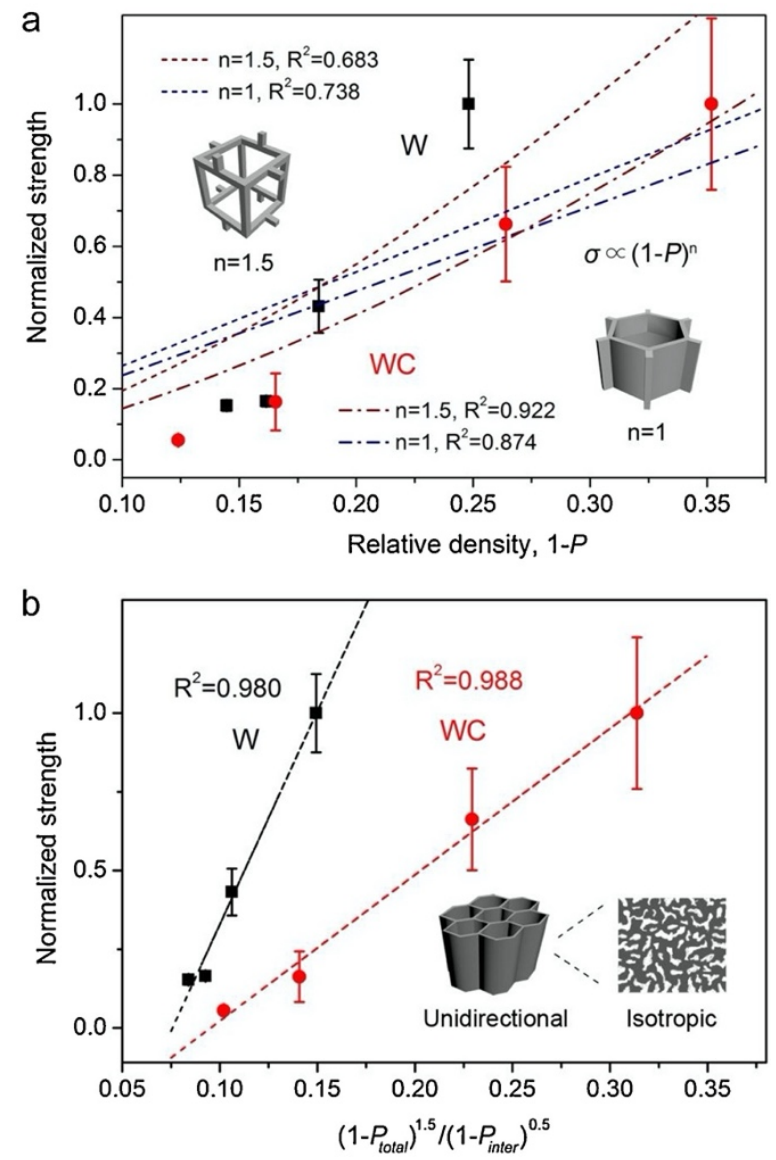

Fig. 9. Relationships between the compressive strengths and porosities for the $W$ and WC scaffolds and the quantitative descriptions (a) following the Gibson-Ashby model for open-cell foams with isotropic and unidirectional pores [54], respectively, and (b) based on the present model by taking both inter-and intra-lamellar pores into account. The insets illustrate the different types of pores involved in the analytical models.

Accordingly, the correlation between the strength and porosity of the scaffolds can be obtained by combining Eqs. (5) and (6) as:

$\sigma / \sigma_{S} \propto\left(1-P_{\text {total }}\right)^{1.5} /\left(1-P_{\text {inter }}\right)^{0.5}$

Li and Dunand have established a similar relationship for ice-templated titanium foams by treating the material as a composite and applying the rule-of-mixtures [55]. By comparison, the present model generates results with a simpler expression and further has clearer physical implications in terms of the mechanics of cellular solids. The strengths of the W and WC scaffolds, normalized by their maxima, depend on their porosities in a similar fashion to that presented in Eq. (7) (Fig. 9(b)), validating the above analysis. Nevertheless, it is noted that the fitted lines do not pass through the origin, but intersect with the vertical axis on the negative side. This implies that the scaffold may become incapable of bearing any load when its porosity exceeds a critical level. The thresholds can be fitted to be, respectively, $\sim 87$ vol.\% and $\sim 89$ vol.\% for $\mathrm{W}$ and WC systems by considering inter-lamellar porosities accounting for, respectively, 
70 vol. $\%$ and 95 vol.\% of the total. Such a trend conforms to the difficulty for the foam to maintain its integrity in the presence of excessive porosity; this is also consistent with the behavior of ice-templated scaffolds with low solid loads where the lamellae are poorly interconnected [56,57].

\subsection{Fracture Mechanisms}

The failure strain, i.e., the strain at the peak stress before fracture, increases with increasing porosity for the W scaffolds (Fig. 10(a)), but displays an opposite trend for the WC system (Fig. 10(b)). This results from their differing fracture mechanisms and specifically the distinctly different effects of porosity (insets of Fig. 10). The lamellae of $\mathrm{W}$ scaffolds become thinner and possess a larger interspace between them as the porosity increases. As such, the deformation, e.g., by bending, and breakage of individual lamellae are much easier to occur at lower stresses, and thus are less prone to cause the failure of the entire scaffolds. This makes the scaffolds capable of undergoing more stable collapse and generate larger nominal plasticity.
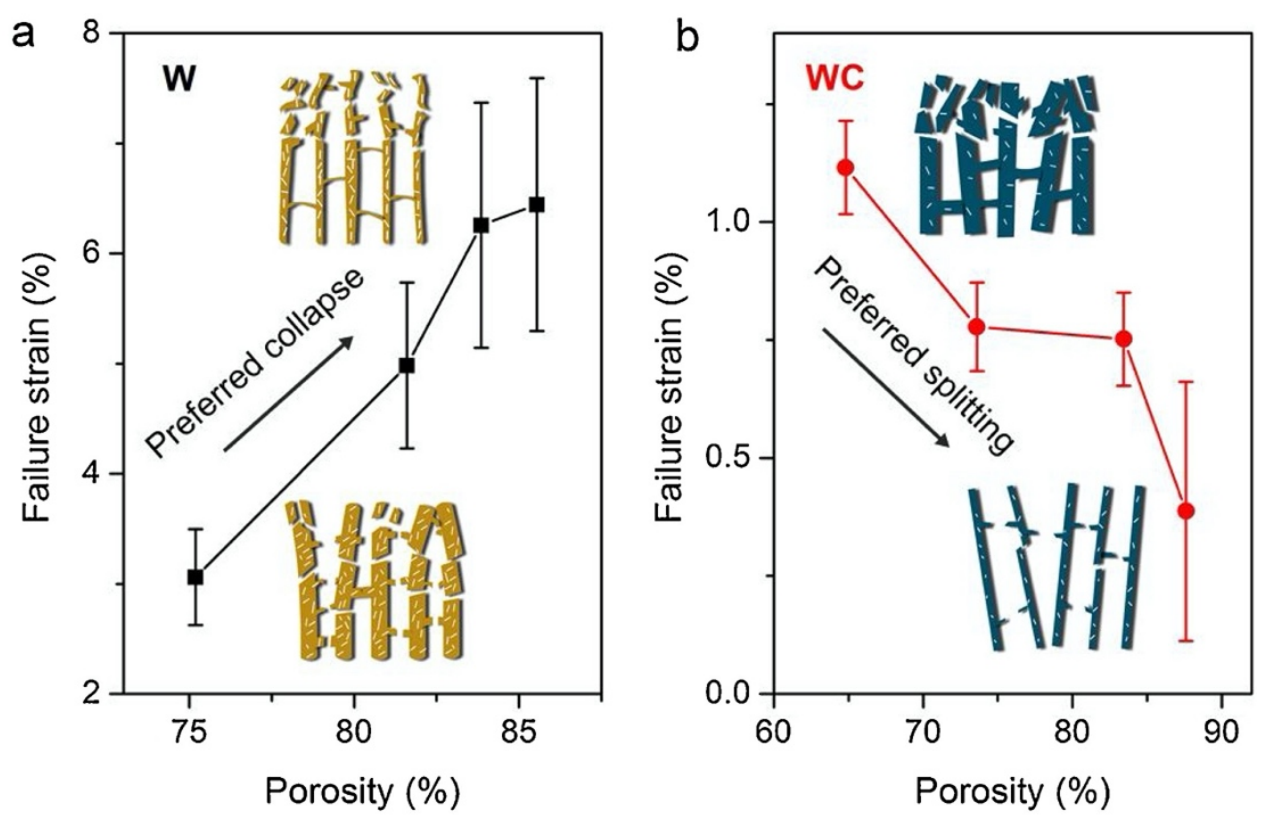

Fig. 10. Variations in the failure strains of the (a) W and (b) WC scaffolds as a function of the total porosity, and schematic illustrations of their fracture mechanisms with the effects of porosity.

By contrast, the separation of lamellae in WC scaffolds with lower porosities becomes increasingly difficult as a result of the enhanced interconnectivity between them. Local rupture of lamellae is thus a more likely scenario without the global splitting fracture of scaffolds (Fig. 8(d)). Moreover, the vertically aligned lamellae display decreased structural ordering in the transverse profile (Fig. S3 in Supplementary Materials), but are intersected with each other. This configuration helps inhibit the extension of splitting cracks throughout the entire scaffolds [56], thereby playing an additional role in retarding the final fracture. 


\subsection{Potential applications}

From a materials science perspective, the unidirectionally porous architecture of wood plays an important role in deriving its extraordinary multi-functionality [[21], [22], [23], [24], [25], [26]]. The replication of such design motif in the present $\mathrm{W}$ and WC scaffolds offers new possibilities towards enhanced properties in their infiltrated composites. This is particularly promising for W/WC-Cu composite systems which can be readily processed by melt infiltration of $\mathrm{Cu}$ into the scaffolds [[2], [3], [4], [5], [6], [7], [8], [9], [10], [11], [12], [13], [14], [15],48,58]. Fig. 11 shows an example of W$\mathrm{Cu}$ composites made by such means; our studies on the processing and properties are currently in progress. The composites feature a lamellar arrangement of constituents which resembles the design motif of nacre, another well-known prototype for biomimetics [18,23,35,48,52,[59], [60], [61], [62]].

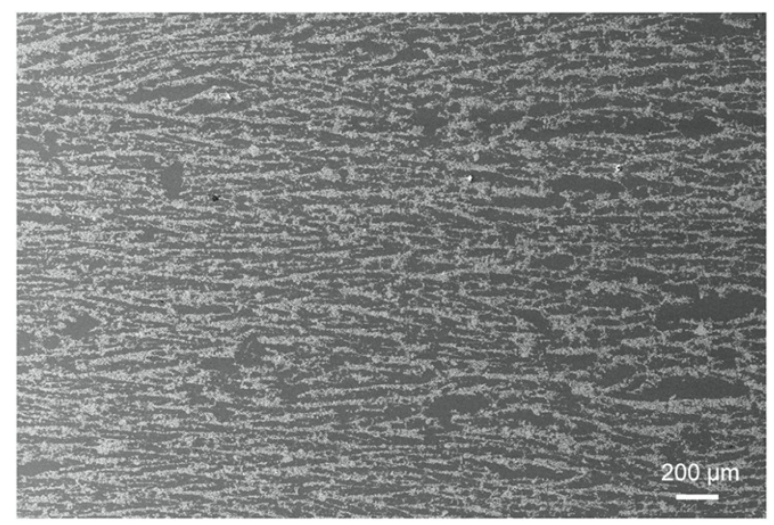

Fig. 11. SEM morphology of a W-Cu composite made by infiltration with a Cu melt into the present $W$ scaffolds. The light and gray phases are $W$ and $C u$, respectively.

The large porosity of over 65 vol.\% in the scaffolds makes it possible to achieve a high $\mathrm{Cu}$ content in the composites exceeding the benchmark for traditional powder metallurgical techniques, i.e., typically lower than $50 \mathrm{vol} . \%$, while still maintaining a good connectivity of individual components. This could improve the thermal and electrical conductivity of the composites and thereby extend their applications. Additionally, the unidirectional architectures may enable the optimization of material performance along specific directions. Specifically, the preferred alignment of W/WC and $\mathrm{Cu}$ is expected to maximize the longitudinal stiffness and conduction properties, and simultaneously lead to good barrier behavior in the transverse section. Moreover, cracks propagating perpendicular to the lamellae are expected to be deflected and/or bridged by uncracked ligaments [35,52,[59], [60], [61], [62]], thereby bestowing enhanced fracture toughness properties in this orientation.

\section{Conclusions}

We have fabricated a suite of scaffolds with differing porosities for W and WC systems by means of direct ice-templating of their stable suspensions containing varying solid 
loads. These scaffolds possess unidirectionally porous architectures which in many respects replicate the design protocol of wood; they represent promising preforms for the development of new multifunctional nacre-like materials such as W/WC-Cu composites. Based on a systematic investigation focused on the processing, microstructures, and mechanical properties of these scaffolds, the following conclusions can be made:

(1) The sedimentation of W/WC powders can be effectively retarded by adjusting the viscosities of suspensions, specifically using HPMC as a thickener. These viscosities increase markedly with increasing HPMC content following a power-law relationship. This allows for the direct ice-templating of W/WC and other material systems with high densities and large particle sizes.

(2) The WC scaffolds display a more ordered alignment of lamellae than for the W system. An increase in solid load leads to the thickening of lamellae and enhanced interconnectivity between them, along with reduced porosities and pore aspect ratios. This is accompanied by the dendritic-to-isotropic and lamellar-to-dendritic transitions of structural morphologies in W and WC systems.

(3) The mechanical properties of these scaffolds are dependent on both the inter- and intra-lamellar pores which are, respectively, unidirectional and isotropic. A strengthporosity relationship, established by integrating their effects in terms of the mechanics of cellular solids, can well describe the experimental results. The scaffolds are deemed to become too weak to withstand any load for the case of excessive porosity over a specific critical level.

(4) The W and WC scaffolds exhibit distinctly different fracture mechanisms in the form of progressive collapse $v s$. brittle splitting. The increase in porosity promotes the collapse of individual lamellae without inducing global fracture for the W system, but causes easy splitting between lamellae for the WC system, thereby resulting in opposing variations in their failure strains.

\section{Acknowledgments}

This work was financially supported by the National Natural Science Foundation of China (Grant Nos. 51871216 and 51501190) and the Opening Project of Jiangsu Province Key Laboratory of High-end Structural Materials (Grant No. hsm1801). Support for R.O.R. was provided by the U.S. Air Force Office of Scientific Research, under MURI grant AFSOR-FA9550-15-1-0009 to the University of California Riverside through a subcontract to the University of California Berkeley.

\section{Competing interests statement}

The authors declare no conflict of interests, financial or otherwise.

\section{Appendix A. Supplementary data}

\section{References}


[1] G.D. Rieck, Tungsten and Its Compounds, first ed., Pergamon Press, Headington Hill Hall, Oxford, UK, 1967.

[2] V. Behrens, W. Weise, Contact materials, in: Landolt-Börnstein-Group VIII Advanced Materials and Technologies (Powder Metallurgy Data), SpringerVerlag Berlin Heidelberg, Berlin, 2003.

[3] F.T.N. Vüllers, R. Spolenak, From solid solutions to fully phase separated interpenetrating networks in sputter deposited "immiscible" W-Cu thin films, Acta Mater. 99 (2015) 213-227.

[4] Y.D. Kim, N.L. Oh, S.T. Oh, I.H. Moon, Thermal conductivity of W-Cu composites at various temperatures, Mater. Lett. 51 (2001) 420-424.

[5] A. Ibrahim, M. Abdallah, S.F. Mostafa, A.A. Hegazy, An experimental investigation on the W-Cu composites, Mater. Design 30 (2009) 1398-1403.

[6] M. Ahangarkani, K. Zangeneh-madar, Investigation on the microstructure and properties of $\mathrm{W}-10 \mathrm{wt} . \% \mathrm{Cu}$ prepared by sintering and infiltration, Int. J. Refract. Metals Hard Mater. 75 (2018) 1-9.

[7] X. Gao, H.Y. Yue, E.J. Guo, S.L. Zhang, L.H. Yao, X.Y. Lin, B. Wang, E.H. Guan, Tribological properties of copper matrix composites reinforced with homogenously dispersed graphene nanosheets, J. Mater. Sci. Technol. 34 (2018) 1925-1931.

[8] O. Ozer, J.-M. Missiaen, S. Lay, R. Mitteau, Processing of tungsten/copper materials from W-CuO powder mixtures, Mater. Sci. Eng. A 460-461 (2007) $525-531$.

[9] A.V. Müller, D. Ewert, A. Galatanu, M. Milwich, R. Neu, J.Y. Pastor, U. Siefken, E. Tejado, J.H. You, Melt infiltrated tungsten-copper composites as advanced heat sink materials for plasma facing components of future nuclear fusion devices, Fusion Eng. Des. 124 (2017) 455-459.

[10] D.I. Tishkevich, S.S. Grabchikov, S.B. Lastovskii, S.V. Trukhanov, D.S. Vasin, T.I. Zubar, A.L. Kozlovskiy, M.V. Zdorovets, V.A. Sivakov, T.R. Muradyan, A.V. Trukhanov, Function composites materials for shielding applications: correlation between phase separation and attenuation properties, J. Alloys Compd. 771 (2019) 238-245.

[11] E. Tejado, A.V. Müller, J.H. You, J.Y. Pastor, The thermo-mechanical behavior of W-Cu metal matrix composites for fusion heat sink applications: the influence of the $\mathrm{Cu}$ content, J. Nucl. Mater. 498 (2018) 468-475.

[12] M. Dias, F. Guerreiro, E. Tejado, J.B. Correia, U.V. Mardolcar, M. Coelho, T. Palacios, J.Y. Pastor, P.A. Carvalho, E. Alves, WC-Cu thermal barriers for fusion applications, Surf. Coat. Tech. 355 (2018) 222-226.

[13] E. Tejado, M. Dias, J.B. Correia, T. Palacios, P.A. Carvalho, E. Alves, J.Y. Pastor, New WC-Cu thermal barriers for fusion applications: high temperature mechanical behavior, J. Nucl. Mater. 498 (2018) 355-361. 
[14] E. Ma, Alloys created between immiscible elements, Prog. Mater. Sci. 50 (2005) 413-509.

[15] L.C. Zhuo, Z. Zhao, Z.C. Qin, Q.Y. Chen, S.H. Liang, X. Yang, F. Wang, Enhanced mechanical and arc erosion resistant properties by homogenously precipitated nanocrystalline fcc- $\mathrm{Nb}$ in the hierarchical $\mathrm{W}-\mathrm{Nb}-\mathrm{Cu}$ composite, Compos. B Eng. 161 (2019) 336-343.

[16] E. Lassner, W. Schubert, Tungsten Properties, Chemistry, Technology of The Element, Alloys, and Chemical Compounds, Springer, Berlin, 1999.

[17] M. Eder, S. Amini, P. Fratzl, Biological composites - complex structures for functional diversity, Science 362 (2018) 543-547.

[18] P.Y. Chen, J. McKittrick, M.A. Meyers, Biological materials: functional adaptations and bioinspired designs, Prog. Mater. Sci. 57 (2012) 1492-1704.

[19] A.R. Studart, Biological and bioinspired composites with spatially tunable heterogeneous architectures, Adv. Funct. Mater. 23 (2013) 4423-4436.

[20] U.G.K. Wegst, M.F. Ashby, The mechanical efficiency of natural materials, Philos. Mag. 26 (2003) 2167-2186.

[21] R. Weinkamer, P. Fratzl, Mechanical adaptation of biological materials - the examples of bone and wood, Mater. Sci. Eng. C 31 (2011) 1164-1173.

[22] J. Keckes, I. Burgert, K. Frühmann, M. Müller, K. Kölln, M. Hamilton, M. Burghammer, S.V. Roth, S. Stanzl-Tschegg, P. Fratzl, Cell-wall recovery after irreversible deformation of wood, Nat. Mater. 2 (2003) 810-813.

[23] Z.Q Liu, M.A. Meyers, Z.F. Zhang, R.O. Ritchie, Functional gradients and heterogeneities in biological materials: design principles, functions, and bioinspired applications, Prog. Mater. Sci. 88 (2017) 467-498.

[24] F. Barthelat, Z. Yin, M.J. Buehler, Structure and mechanics of interfaces in biological materials, Nat. Rev. Mater. 1 (2016) 1-16.

[25] T. Li, Y. Zhai, S. He, W. Gan, Z. Wei, M. Heidarinejad, D. Dalgo, R. Mi, X. Zhao, J. Song, J. Dai, C. Chen, A. Aili, A. Vellore, A. Martini, R. Yang, J. Srebric, X. Yin, L. Hu, A radiative cooling structural material, Science 364 (2019) 760-763.

[26] T. Speck, I. Burgert, Plant stems: functional design and mechanics, Annu. Rev. Mater. Res. 41 (2011) 169-193.

[27] N. Sarkar, Thermal gelation properties of methyl and hydroxypropyl methylcellulose, J. Appl. Polym. Sci. 24 (1979) 1073-1087.

[28] H. Bai, Y. Chen, B. Delattre, A.P. Tomsia, R.O. Ritchie, Bioinspired large-scale aligned porous materials assembled with dual temperature gradients, Sci. Adv. 1 (2015) e1500849.

[29] S. Deville, E. Saiz, A.P. Tomsia, Ice-templated porous alumina structures, Acta Mater. 55 (2007) 1965-1974. 
[30] D. Ghosh, N. Dhavale, M. Banda, H. Kang, A comparison of microstructure and uniaxial compressive response of ice-templated alumina scaffolds fabricated from two different particle sizes, Ceram. Inter. 42 (2016) 16138-16147.

[31] T.P. Carr, D.D. Gallaher, C.H. Yang, C.A. Hassel, Increased intestinal contents viscosity reduces cholesterol absorption efficiency in hamsters fed Hydroxypropyl methylcellulose, J. Nutr. 126 (1996) 1463-1469.

[32] M. Mooney, The viscosity of a concentrated suspension of spherical particles, J. Colloid Sci. 6 (1951) 162-170.

[33] G.G. Stokes, On the effect of the internal friction of fluids on the motion of pendulums, Trans. Cambridge Phil. Soc. 9 (1851) 8-106.

[34] V. Naglieri, H.A. Bale, B. Gludovatz, A.P. Tomsia, R.O. Ritchie, On the development of ice-templated silicon carbide scaffolds for nature-inspired structural materials, Acta Mater. 61 (2013) 6948-6957.

[35] V. Naglieri, B. Gludovatz, A.P. Tomsia, R.O. Ritchie, Developing strength and toughness in bio-inspired silicon carbide hybrid materials containing a compliant phase, Acta Mater. 98 (2015) 141-151.

[36] A. Röthlisberger, S. Häberli, R. Spolenak, D.C. Dunand, Synthesis, structure and mechanical properties of ice-templated tungsten foams, J. Mater. Res. 31 (2016) 753-764.

[37] S. Deville, E. Saiz, R.K. Nalla, A.P. Tomsia, Freezing as a path to build complex composites, Science 311 (2006) 515-518.

[38] P.M. Hunger, A.E. Donius, U.G.K. Wegst, Structure-property-processing correlations in freeze-cast composite scaffolds, Acta Biomater. 9 (2013) 63386348.

[39] K.L. Scotti, D.C. Dunand, Freeze casting - a review of processing, microstructure and properties via the open data repository, FreezeCasting.net, Prog. Mater. Sci. 94 (2018) 243-305.

[40] S. Deville, Freeze-casting of porous biomaterials: structure, properties and opportunities, Materials 3 (2010) 1913-1927.

[41] C. Ferraro, S. Meille, J. Rethore, N. Ni, J. Chevalier, Strong and tough metal/ceramic micro-laminates, Acta Mater. 144 (2018) 202-215.

[42] X.M. Liu, N. Chai, Z.J. Yu, H.L. Xu, X.L. Li, J.Q. Liu, X.W. Yin, R. Riedel, Ultra-light, high flexible and efficient $\mathrm{CNTs} / \mathrm{Ti}_{3} \mathrm{C}_{2}$-sodium alginate foam for electromagnetic absorption application, J. Mater. Sci. Technol. doi: 10.1016/j.jmst.2019.07.019.

[43] S. Deville, E. Saiz, A.P. Tomsia, Freeze casting of hydroxyapatite scaffolds for bone tissue engineering, Biomaterials 27 (2006) 5480-5489.

[44] U.G.K. Wegst, M. Schecter, A.E. Donius, P.M. Hunger, Biomaterials by freeze casting, Phil. Trans. R. Soc. A 368 (2010) 2099-2121. 
[45] Y. Chino, D.C. Dunand, Directionally freeze-cast titanium foam with aligned, elongated pores, Acta Mater. 56 (2008) 105-113.

[46] P.W. Style, S.S.L. Peppin, The kinetics of ice-lens growth in porous media, J. Fluid Mech. 692 (2012) 482-498.

[47] A. Lasalle, C. Guizard, E. Maire, J. Adrien, S. Deville, Particle redistribution and structural defect development during ice templating, Acta Mater. 60 (2012) 4594-4603.

[48] A. Röthlisberger, S. Häberli, F. Krogh, H. Galinski, D.C. Dunand, R. Spolenak, Ice-templated W-Cu composites with high anisotropy, Sci. Rep. 9 (2019) 1-9.

[49] Y.S. Lee, S.T. Oh, Fabrication and properties of porous tungsten by freezedrying process, Kor. J. Mater. Res. 21 (2011) 520-524.

[50] H. Park, H.H Cho, K. Kim, K. Hong, J.H. Kim, H. Choe, D.C. Dunand, Surfaceoxidized, freeze-cast cobalt foams: microstructure, mechanical properties and electrochemical performance, Acta Mater. 142 (2018) 213-225.

[51] S.K. Wilke, D.C. Dunand, Structure evolution of directionally freeze-cast iron foams during oxidation/reduction cycles, Acta Mater. 162 (2019) 90-102.

[52] F. Bouville, E. Maire, S. Meille, B.V. Moortèle, A.J. Stevenson, S. Deville, Strong, tough and stiff bioinspired ceramics from brittle constituents, Nat. Mater. 13 (2014) 508-214.

[53] R. Asthana, S.N. Tewari, The engulfment of foreign particles by a freezing interface, J. Mater. Sci. 28 (1993) 5414-5425.

[54] L.J. Gibson, M.F. Ashby, Cellular Solid: Structure and Properties, second ed., Cambridge University Press, Cambridge, UK, 1999.

[55] J.C. Li, D.C. Dunand, Mechanical properties of directionally freeze-cast titanium foams, Acta Mater. 59 (2011) 146-158.

[56] J. Seuba, S. Deville, C. Guizard, A.J. Stevenson, The effect of wall thickness distribution on mechanical reliability and strength in unidirectional porous ceramics, Sci. Technol. Adv. Mater. 17 (2016) 128-135.

[57] A. Lichtner, D. Roussel, D. Jauffres, C.L. Martin, R.K. Bordia, Effect of macropore anisotropy on the mechanical response of hierarchically porous ceramics, J. Am. Ceram. Soc. 99 (2016) 979-987.

[58] M. Calvo, A.E. Jakus, R.N. Shah, R. Spolenak, D.C. Dunand, Microstructure and processing of $3 \mathrm{D}$ printed tungsten microlattices and infiltrated $\mathrm{W}-\mathrm{Cu}$ composite, Adv. Eng. Mater. 20 (2018) 1-9.

[59] D. Jiao, Z.Q. Liu, Y.K. Zhu, Z.Y. Weng, Z.F. Zhang, Mechanical behavior of mother-of-pearl and pearl with flat and spherical laminations, Mater. Sci. Eng. C 68 (2016) 9-17.

[60] U.G.K. Wegst, H. Bai, E. Saiz, A.P. Tomsia, R.O. Ritchie, Bioinspired structural materials, Nat. Mater. 14 (2015) 23-36. 
[61] M.E. Launey, E. Munch, D.H. Alsem, E. Saiz, A.P. Tomsia, R.O. Ritchie, A novel biomimetic approach to the design of high-performance ceramic-metal composites, J. R. Soc. Interface 7 (2010) 741-753.

[62] M.Y. Zhang, D. Jiao, G.Q. Tan, J. Zhang, S.G. Wang, J.Y. Wang, Z.Q. Liu, Z.F. Zhang, R.O. Ritchie, Strong, fracture-resistant biomimetic silicon carbide composites with laminated interwoven nanoarchitectures inspired by the crustacean exoskeleton, ACS Appl. Nano. Mater. 2 (2019) 1111-1119. 